\title{
Status of the NEOS experiment
}

\author{
Yoomin. Oh ${ }^{*}{ }^{a}$ B. Y. Han, ${ }^{b}$ C. H. Jang, ${ }^{c}$ E. J. Jeon, ${ }^{a}$ K. K. Joo, ${ }^{d}$ B. R. Kim, ${ }^{d}$ H. J. Kim, ${ }^{e}$

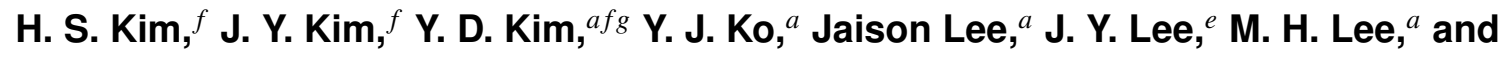 \\ H. K. Park ${ }^{h}$ \\ ${ }^{a}$ Center for Underground Physics, Institute for Basic Science (IBS), Deajeon 34126, Korea \\ ${ }^{b}$ Neutron Science Division, Korea Atomic Energy Research Institute, Deajeon 34057, Korea \\ ${ }^{c}$ Department of Physics, Chung-Ang University, Seoul 06974, Korea \\ ${ }^{d}$ Department of Physics, Chonnam National University, Gwangju 61186, Korea \\ ${ }^{e}$ Department of Physics, Kyungpook National University, Daegu 41566, Korea \\ ${ }^{f}$ Department of Physics, Sejong University, Seoul 05006, Korea \\ ${ }^{g}$ University of Science and Technology, Daejeon 34113, Korea \\ ${ }^{h}$ Department of Accelerator Science, Korea University, Sejong 30019, Korea
}

The NEOS experiment has successfully measured the reactor antineutrino energy spectrum at $24 \mathrm{~m}$ distance from Hanbit reactor unit 5 for 180 days of reactor operation and constrained the active-to-sterile oscillation parameters. An extended measurement for a whole burnup cycle by the NEOS Phase-II will be a unique probe for the dependence of the reactor antineutrino flux and spectrum on the fuel composition.

The 39th International Conference on High Energy Physics (ICHEP 2018)

4-11 July, 2018

Seoul, Korea

* Speaker. 
A number of experiments have been being performed or are being planned to be performed at various distances from nuclear reactors, which are powerful sources of electron antineutrinos. Several neutrino mixing parameters have been measured at mid- and long-baseline experiments $[1,2,3,4]$. On the other hand, a so-called Reactor Antineutrino Anomaly (RAA) has been reported [5] from the compilation of the results of various short baseline experiments including the reactor experiments at 10-100 m distances from the active core. The anomaly is that the average of measured antineutrino flux is about $6 \%$ less than theoretically expected with about $2.6 \sigma$ significance level. This deficit, combined with the anomalies from accelerator experiments, can be explained by adding another mass and flavor state to the existing 3 neutrino mass and flavor framework. The fourth flavor state involves no standard electroweak interaction and thus is called "sterile". And the RAA favors the fourth mass state at around eV scale.

In the meanwhile, questions arise on the theoretical prediction of the reactor antineutrino flux. The three mid-baseline experiments [1,2,3] have reported similar disagreement between measured and predicted energy spectra, especially at around $5 \mathrm{MeV}$ in the prompt energy . And the Daya Bay experiment measured the changes of the flux and spectrum along with the fission fractions' changes of U-235 and Pu-239 elements, and claimed that the miscalculated antineutrino flux for U-235 in the prediction model can be the reason for the RAA [6]. However, measured fluxes for other fission elements still have large uncertainties to exclude the eV sterile neutrino scenario.

Recently, experiments focusing on detection of the active-to-sterile neutrino oscillation are being performed at several places over the world. Since most of these short baseline experiments are conducted with lack of overburden in limited spaces, they suffer from shortage of shielding materials and, thus, high background event rate. Various techniques such as segmentation of the active volume of the detector, enhancing the inverse-beta-decay (IBD) neutron capture efficiency, and pulse shape discrimination, have been developed and selectively applied to each experiment. In addition, to overcome the uncertainties in the theoretical reactor flux models, experiments try to have their own baseline resolutions by moving the detector and/or by segmented design.

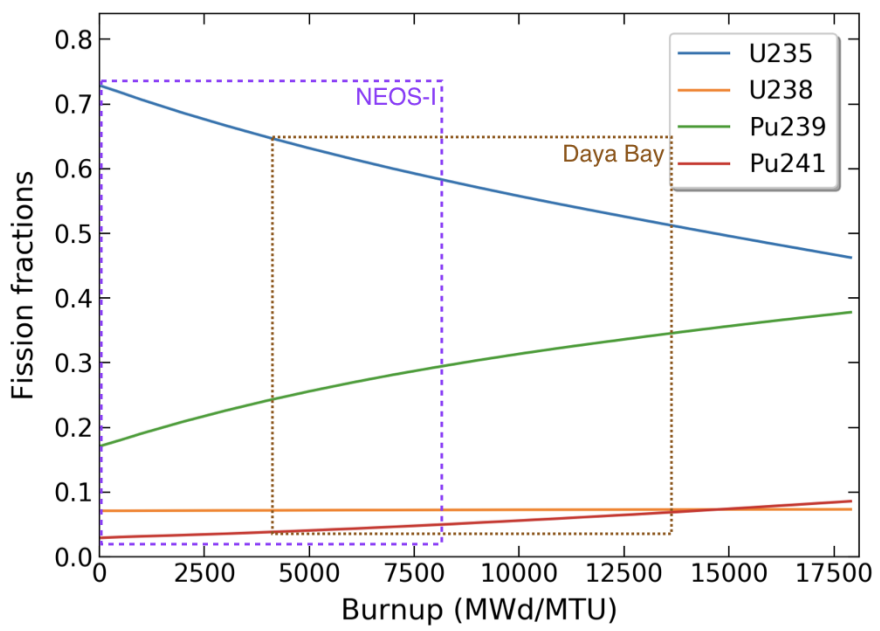

Figure 1: A typical fission fractions and their changes during one operation cycle of Hanbit-5 reactor. NEOS measured the data for about first half of an operation cycle during phase-I measurement. Daya Bay [6] can measure only average of several reactors. The next phase of NEOS will measure the full operation cycle and will see the effects of larger fission fraction changes.

NEOS, standing for Neutrino Oscillation at Short baseline, uses the Hanbit-5 reactor located in Yeonggwang, Korea. The commercial reactor has $2.8 \mathrm{GW}$ thermal power and emits about $6 \times 10^{20}$ 
electron antineutrinos per second. A typical fission fractions of primary fissile elements in the low-enriched uranium fuel for an operation cycle is shown in Fig. 1. The detector is located in the tendon gallery of the containment, where at least 20 meter water equivalent overburden is provided. The distance between the reactor core and the detector center is $23.7 \mathrm{~m}$. The antineutrino detector consists of $1000 \mathrm{~L}$ of homogeneous $0.5 \%$ Gd-loaded liquid scintillator (Gd-LS), layers of passive shielding of $10 \mathrm{~cm}$ thick borated polyethylene and $10 \mathrm{~cm}$ thick $\mathrm{Pb}$, and a layer of plastic scintillator for the active muon counter. The Gd-LS is in the horizontal cylindrical stainless tank and seen by 2 sets of nineteen 8-inch phototubes on the two endcaps, of which the waveforms are processed through $500 \mathrm{MHz}$ sampling analog-to-digital converters. A mixing of 10\% di-isopropylnaphthalene based LS to the linear alkylbenzene based LS enhanced the pulse shape discrimination power for distinguishing the fast neutrons' elastic scattering events from electron recoil events.

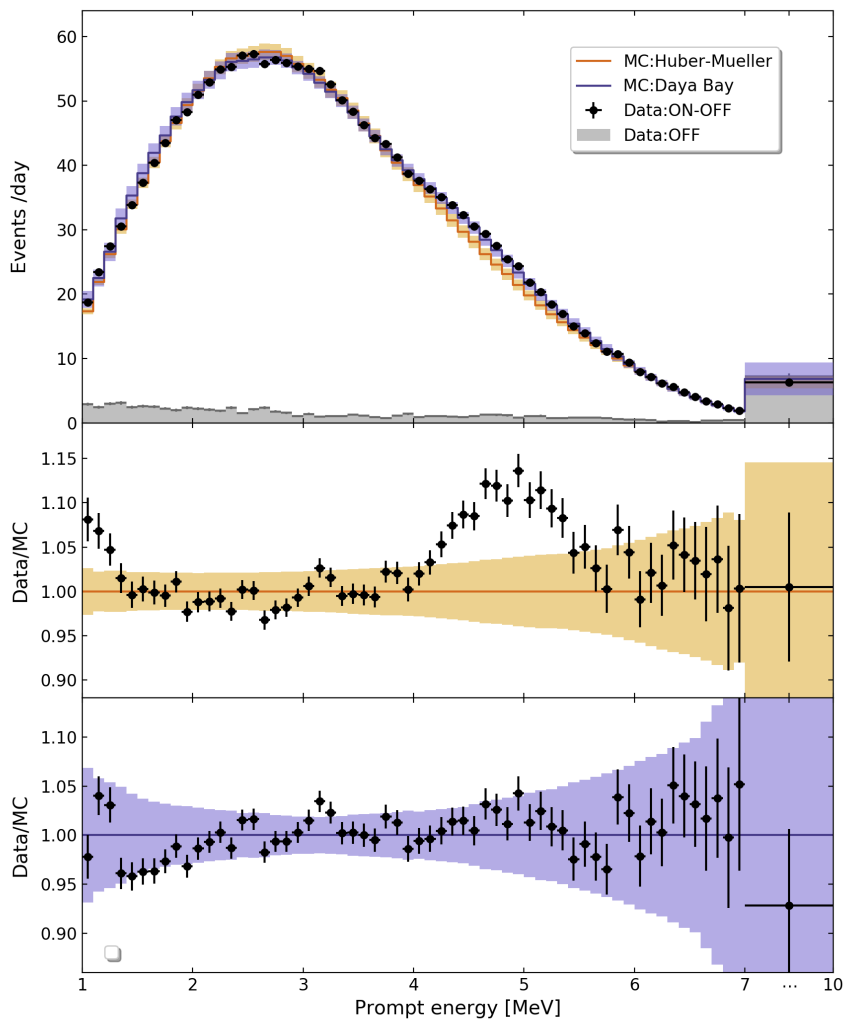

Figure 2: Measured IBD prompt energy spectrum (black dots in top), and its ratio to the reference spectra (in middle and bottom panels): one generated from the Huber's and Mueller's flux models $[7,8]$ and Vogel's cross section [9] (Orange in middle), and the other from the Daya Bay's absolute measurement result [10] (dark violet in bottom). Background spectrum measured during reactor-off period is denoted as gray histogram in the top panel.

The analysis result of the first phase of the measurement including 46 livedays of reactor-off period data and 180 livedays of reactor-on period data is in Ref. [11]. A comparison of the spectral shape between the measured IBD prompt energy spectrum and a reference from the Daya Bay's result [10] showed no strong evidence of active-to-sterile neutrino oscillation for $\Delta m_{41}^{2} \sim 1 \mathrm{eV}$. The other comparison between the measured spectrum and the theoretical calculation showed a disagreement at around $5 \mathrm{MeV}$ prompt energy range, similar with the result from the three midbaseline experiments. In both ratio plots of Fig. 2, small fluctuations were observed not only at around $5 \mathrm{MeV}$ region, but several other ranges. This may imply that, unless those fluctuations are 
not from any oscillation, the reactor neutrino spectrum is not so smooth as expected [12] and to check this, a measurement with a good energy resolutions is necessary.

For further utilizations of the reactor antineutrino sources, uncertainties in their properties, especially in the flux and energy spectrum from each must be resolved. NEOS has a unique opportunity to measure the spectrum with the largest differences in the fission fractions of primary fissile elements in a reactor operation cycle (Fig. 1). With almost twice larger changes in the fission fractions, the NEOS phase-II measurement is expected to show a similar statistical uncertainty level in the flux and spectrum evolution with the Daya Bay's result [6]. Decomposition of the spectrum for ${ }^{235} \mathrm{U}$ and ${ }^{239} \mathrm{Pu}$ will be also interesting to probe the origin of the spectral anomaly. The phase-II measurement is scheduled to start from September 2018, and will be conducted for about 2 years, measuring about 500 days of reactor-on data and two reactor-off periods' data before and after the operation cycle.

\section{References}

[1] Daya BAy collaboration, F. P. An et al., Phys. Rev. Lett. 116 (2016) 061801.

[2] RENO collaboration, J. H. Choi et al., Phys. Rev. Lett. 116 (2016) 211801.

[3] Double Chooz collaboration, Y. Abe et al., Phys. Rev. D86 (2012) 052008.

[4] KamLAND collaboration, K. Eguchi et al., Phys. Rev. Lett. 90 (2003) 021802.

[5] G. Mention, M. Fechner, T. Lasserre, T. A. Mueller, D. Lhuillier, M. Cribier et al., Phys. Rev. D83 (2011) 073006.

[6] Daya Bay collaboration, F. P. An et al., Phys. Rev. Lett. 118 (2017) 251801.

[7] P. Huber, Phys. Rev. C84 (2011) 024617

[8] T. A. Mueller et al., Phys. Rev. C83 (2011) 054615.

[9] P. Vogel and J. F. Beacom, Phys. Rev. D60 (1999) 053003.

[10] DAYA BAY collaboration, F. P. An et al., Chin. Phys. C41 (2017) 013002.

[11] NEOS collaboration, Y. Ko et al., Phys. Rev. Lett. 118 (2017) 121802.

[12] A. A. Sonzogni, M. Nino and E. A. McCutchan, Phys. Rev. C98 (2018) 014323. 